\title{
EFEITO DO ULTRASSOM TERAPÊUTICO SOBRE AS PROPRIEDADES MECÂNICAS DO GASTROCNÊMIO EM RATOS
}

\author{
EFFECT OF THERAPEUTIC ULTRASOUND ON MECNANICAL PROPERTIES \\ OF GASTROCNEMIUS IN RATS
}

\author{
EFECTO DEL ULTRASOUNIDO TERAPÉUTICO SOBRE LAS PROPIEDADES MECÁNICAS \\ DEL GASTROCNEMIO EN RATONES
}

\author{
Hállamo Henrique Saraiva \\ Barbosa' (Fisioterapeuta) \\ José Humberto do Nascimento \\ Filho ${ }^{1}$ (Fisioterapeuta) \\ Dayanne Terra Tenório Nonato \\ (Fisioterapeuta) \\ Magno Jackson Moreno de \\ Almeida' (Fisioterapeuta) \\ Flávio Santos Silva ${ }^{2}$ \\ (Fisioterapeuta) \\ Bento João $\mathrm{Abreu}^{3}$ \\ (Fisioterapeuta) \\ Wouber Hérickson de Brito Vieira ${ }^{4}$ \\ (Fisioterapeuta) \\ 1. Curso de Fisioterapia da \\ Universidade Potiguar, Natal, Rio \\ Grande do Norte, Brasil. \\ 2. Universidade Federal do Rio \\ Grande do Norte, Natal, Rio \\ Grande do Norte, Brasil. \\ 3. Departamento de Morfologia, \\ Centro de Biociências da \\ Universidade Federal do Rio \\ Grande do Norte, Rio Grande do \\ Norte, Natal, Brasil. \\ 4. Departamento de Fisioterapia, \\ Centro de Ciências da Saúde \\ da Universidade Federal do Rio \\ Grande do Norte, Rio Grande do \\ Norte, Natal, Brasil.
}

\section{Correspondência:}

Wouber Hérickson de Brito Vieira, Departamento de Fisioterapia / Universidade Federal do Rio Grande do Norte. Av. Senador Salgado Filho, 3000 - Campus Universitário Lagoa Nova, Natal. RN, Brasil. 59072-970. hericksonfisio@yahoo.com.br

\begin{abstract}
RESUMO
Introdução: Apesar de algumas controvérsias quanto à sua utilização, o ultrassom terapêutico (UST) é um recurso comumente aplicado na reabilitação desportiva para aceleração do reparo tecidual de lesões musculares. Sabe-se que lesões musculares influenciam negativamente as propriedades mecânicas da musculatura estriada esquelética e algumas evidências demonstram que o UST poderia ter efeitos benéficos sobre o reparo muscular e, consequentemente, sobre suas propriedades mecânicas. Objetivo: Analisar o efeito do UST no reparo tecidual por meio das propriedades mecânicas musculares de ratos após trauma por criolesão. Métodos: Foram utilizados no estudo 30 ratos da linhagem Wistar, os quais foram divididos em três grupos: grupo controle intacto (GC), grupo lesionado sem tratamento (GL), e grupo lesionado e estimulado com UST (frequência de $1 \mathrm{MHz}$; intensidade de $0,5 \mathrm{~W} / \mathrm{cm}^{2}$; ciclo de trabalho de 50\%; por quatro minutos diários), durante sete dias consecutivos (GLUST). As propriedades mecânicas obtidas através de ensaio mecânico de tração foram avaliadas em uma máquina universal de ensaios. Resultados: Foram analisados estatisticamente, com nível de significância de 95\% ( $P<0,05)$. Após sete sessões de tratamento, houve melhora estatisticamente significativa nas propriedades mecânicas de carga no limite de proporcionalidade, carga no limite máximo e resiliência para o GLUST em relação ao GL $(p<0,05)$. Conclusão: O UST foi eficaz no processo de reparo tecidual, conferindo ao tecido muscular maior resistência à tração e absorção de energia.
\end{abstract}

Palavras-chave: terapia por ultrassom, musculoesquelético, biomecânica.

\begin{abstract}
Introduction: Despite controversial, ultrasonic therapy (UST) is commonly applied in sports rehabilitation practice to accelerate tissue repair after muscle damage. Muscle injuries have a negative influence on the mechanical properties of skeletal striated muscles and some scientific evidence shows that UST could have a positive effects on tissue repair and, therefore, on its mechanical properties. Objective: To analyze the effect of UST on muscle repair by evaluation of the mechanical properties of rat muscles after cryolesion. Methods: We used 30 adult Wistar rats which were divided into three groups: CG (control group), IG (injured group) and IGUST (injured plus UST) - (frequency of $1 \mathrm{MHz}$; intensity of $0.5 \mathrm{~W} / \mathrm{cm}^{2}$; pulsed to 50\%; four minutes daily) for seven consecutive days. The mechanical properties obtained through mechanical tests were evaluated in a universal testing machine. Results: Data were statistically analyzed with a significance level of $95 \%(p<0.05)$. After seven treatment sessions, we found significant improvement in the mechanical properties of load at the proportionality limit, load at maximum limit and resilience in IGUST compared to IG ( $p<0.05)$. Conclusion: The UST was effective in the tissue repair process, giving higher tensile strength and energy absorption to the muscle tissue.
\end{abstract}

Keywords: ultrasonic therapy, muscle, skeletal, biomechanics.

\section{RESUMEN}

Introducción: A pesar de algunas controversias cuanto a su utilización, el ultrasonido terapéutico (UST) es un recurso aplicado comúnmente en la rehabilitación deportiva para aceleración de la reparación tecidual de lesiones musculares. Se sabe que las lesiones musculares influyen negativamente en las propiedades mecánicas de la musculatura estriada esquelética y algunas evidencias demuestran que el UST podría tener efectos sobre la reparación muscular y, en consecuencia, con respecto a sus propiedades mecánicas. Objetivo: Analizar el efecto del UST en la reparación tecidual por medio de las propiedades mecánicas musculares de ratones después de traumatismo por criolesión. Métodos: Se utilizaron, en el estudio, 30 ratones de la raza Wistar, los cuales fueron divididos en tres grupos: grupo de control intacto (GC), grupo lesionado sin tratamiento (GL) y grupo lesionado y estimulado con UST (frecuencia de $1 \mathrm{MHz}$; intensidad de 0,5 W/cm²; ciclo de trabajo de 50\%, durante cuatro minutos diarios) a 10 largo de siete días consecutivos (GLUST). Las propiedades mecánicas obtenidas, mediante el ensayo mecánico de 
tracción, fueron evaluadas en una máquina universal de ensayos. Resultados: Se analizaron estadísticamente, con nivel de significancia de 95\% ( $P<0,05)$. Después de siete sesiones de entrenamiento, hubo mejora estadísticamente significativa en las propiedades mecánicas de carga en el límite de proporcionalidad, carga en el límite máximo y resiliencia para el GLUST en relación con el GL $(p<0,05)$. Conclusión: El UST fue eficaz en el proceso de reparación tecidual, dando al tejido muscular más resistencia cuanto a la tracción y la absorción de energía.

Palabras clave: terapia por ultrasonido, músculo esquelético, biomecánica.

\section{INTRODUÇÃO}

As lesões musculares são os acometimentos mais comuns encontrados no esporte e podem responder por até 50\% das lesões ${ }^{1}$. Devido à heterogeneidade na severidade e local das lesões, seu tratamento é considerado um desafio para diversos profissionais que lidam diretamente com a reabilitação do atleta profissional ou recreativo².

Algumas modalidades terapêuticas visam promover melhorias no reparo do tecido muscular. Dentre elas, o ultrassom terapêutico (UST) é uma modalidade frequentemente empregada no meio clínico para aceleração do reparo tecidual e para tratamento de diversos distúrbios músculoesqueléticos em atletas tais como dor, contraturas articulares, tendinites, bursites, espasmos e contusões musculares ${ }^{3-8}$.

O UST poderia atuar no processo de regeneração por meio de vibrações acústicas, as quais podem propiciar diversas alterações como aumento da síntese proteica e secreção de mastócitos, modificações na mobilidade dos fibroblastos, dentre outras ${ }^{9}$.

Vários resultados sobre os efeitos de UST podem ser observados em modelos histológicos, e apresentam-se como uma importante ferramenta na avaliação do reparo tecidual ${ }^{10-14}$. Ainda, diferenças entre as fases de reparo tecidual e a própria qualidade do reparo podem ser funcionalmente evidenciadas por meio de ensaios mecânicos de materiais biológicos ${ }^{15}$. Tais testes consistem no alongamento do tecido previamente isolado pela aplicação de uma carga externa até sua ruptura total, onde podem ser registrados diversos parâmetros como: carga no limite de proporcionalidade, alongamento no limite de proporcionalidade, carga no limite máximo, alongamento no limite máximo, rigidez e resiliência ${ }^{16,17}$

No entanto, apesar do uso frequente do UST na prática clínica, sua efetividade permanece questionável ${ }^{18}$. Além disso, poucos trabalhos abordaram o comportamento mecânico de tecidos biológicos sob esta modalidade terapêutica ${ }^{5,19,20}$. Diante disto, o objetivo do estudo foi elucidar o comportamento biomecânico do músculo gastrocnêmio de ratos submetidos à criolesão aguda e tratados com UST.

\section{MATÉRIAIS E MÉTODOS}

Para a realização dos experimentos foram utilizados 30 ratos da linhagem Wistar (Rattos Norvegicos Albinus) com 90 dias, pesando aproximadamente $300 \mathrm{~g}$. Os animais foram alocados em gaiolas coletivas, em ambiente com temperatura e iluminação controladas, onde tiveram livre acesso à água e ração. O presente estudo foi aprovado pelo Comitê de Ética em Pesquisa da Universidade Potiguar, de acordo com o protocolo de número 095/2009.

Os animais foram distribuídos, aleatoriamente, em três grupos compostos por 10 ratos cada um: grupo controle (GC), formado por animais que foram mantidos em gaiolas de contenção por nove dias; grupo lesão muscular sem tratamento (GL), composto por animais que tiveram o músculo gastrocnêmio direito submetido à lesão por congelamento (criolesão) e não passaram por nenhuma intervenção terapêutica. Após a lesão, foram acompanhados por nove dias em suas gaiolas; e grupo lesão muscular tratado com UST (GLUST), formado por animais que sofreram criolesão no músculo gastrocnêmio direito e, após 24 horas, foram submetidos à irradiação por UST de $1 \mathrm{MHz}$ no modo pulsátil de baixa intensidade, durante os sete dias subsequentes.

Previamente à produção da lesão muscular nos animais dos grupos GL e GLUST, foram tomadas as devidas medidas de assepsia do local e dos equipamentos. Em seguida, os animais foram anestesiados com associação de tiletamina e zolazepam na dose de $50 \mathrm{mg} / \mathrm{kg}$. Já anestesiados, induziu-se dano tecidual por criolesão, por meio de um bastão metálico (seis milímetros de largura e altura, por $30 \mathrm{~mm}$ de comprimento), previamente imerso em nitrogênio líquido, o qual foi pressionado transversalmente contra o ventre do músculo gastrocnêmio direito. O contato do bastão metálico com a musculatura foi realizado duas vezes, com duração de 10 segundos cada e intervalo de 10 segundos entre as duas aplicações ${ }^{21}$. Após breve descongelamento, a pele que recobre a lesão foi suturada com fio de seda odontológico 4-0. Vale destacar que esse procedimento foi realizado pelo mesmo pesquisador devidamente treinado para esta tarefa.

O equipamento utilizado para o tratamento do grupo GLUST foi um gerador ultrassônico da marca Bioset ${ }^{\circledR}$, modelo Sonacel Dual, com frequência de $1 \mathrm{MHz}$, devidamente calibrado. Ao equipamento, foi acoplado um cabeçote de diâmetro reduzido $(1 \mathrm{~cm}), \operatorname{com} 0,8 \mathrm{~cm}^{2}$ de área de radiação efetiva ERA, especialmente confeccionado pelo fabricante.

Decorridas 24 horas após a lesão experimental, foi iniciado o tratamento no músculo gastrocnêmio direito destes animais, em sessões diárias, por sete dias consecutivos. Os parâmetros do UST utilizados neste estudo foram: modalidade pulsada com frequência modulada de $100 \mathrm{~Hz}$, durante 4 minutos, com ciclo de trabalho de 50\% (1:1), intensidade de $0,5 \mathrm{~W} / \mathrm{cm}^{2}$ e frequência de $1 \mathrm{MHz}$.

Durante a aplicação da irradiação ultrassônica, foram utilizados movimentos circulares sobre o local da lesão e gel hidrossolúvel como meio de contato entre a interface aparelho-tecido. Esse procedimento terapêutico foi realizado por outro pesquisador previamente treinado na técnica de aplicação.

Após o fim do tratamento, os animais foram sacrificados em uma câmara de $\mathrm{CO} 2$ para que seus músculos gastrocnêmios direitos fossem isolados e submetidos ao ensaio mecânico de tração.

Na dissecação, foram removidas as partes moles adjacentes, preservando o tecido muscular juntamente com o osso calcâneo e parte distal do fêmur, de modo a obter um complexo osso-músculo-osso. Em seguida, os espécimes foram colocados em solução de lactato de Ringer, em temperatura ambiente, por aproximadamente 30 minutos, quando se deram os ensaios.

Previamente à efetivação dos ensaios de tração definitivos, foram realizados testes piloto com os músculos gastrocnêmios das patas esquerdas, no propósito de aprimorar o procedimento de forma global. Esta fase foi importante para padronizar o modo de fixação dos espécimes ao sistema, bem como a velocidade de aplicação da carga utilizada nos ensaios. Uma vez que foi verificada a reprodutibilidade dos resultados piloto, iniciaram-se os testes definitivos.

Os espécimes coletados tiveram suas extremidades ósseas 
(proximal e distal) fixadas a garras metálicas ajustáveis da máquina universal de ensaios. Vale destacar que todos os espécimes foram posicionados do mesmo modo, alinhando o maior eixo das fibras musculares ao eixo de tensão do dispositivo mecânico.

Para a realização dos testes de tração, utilizou-se uma máquina universal de ensaios (modelo AG-1 250kN, SHIMADZU ${ }^{\circledR}$ ), equipada com célula de carga de capacidade de $50 \mathrm{kgf}$. A máquina possui interface direta a um microcomputador, com o software TRAPEZIUM $X^{\circledR}$, capaz de gerar um gráfico carga versus alongamento para cada ensaio. Com o propósito de acomodação do sistema, foi aplicada uma pré-carga de $200 \mathrm{~g}$ durante 30 segundos. Tais procedimentos foram previamente validados em estudo prévio $^{20}$. Finalmente, o ensaio prosseguiu com velocidade de $10 \mathrm{~mm} / \mathrm{min}$. A carga aplicada foi registrada pelo software em intervalos regulares de alongamento até o momento em que o músculo sofreu ruptura.

Assim, foram obtidos gráficos carga versus alongamento a partir dos resultados de cada ensaio, de onde foram extraídas e analisadas as seguintes propriedades mecânicas, de acordo com Polizello et al. ${ }^{17}$ : carga no limite de proporcionalidade, cujo valor identifica a carga suportada no limite elástico (registrado em Newtons, N); alongamento no limite de proporcionalidade, que é o valor de alongamento exibido no limite elástico (em milímetros, mm); carga no limite máximo, que representa o maior valor de carga registrado na fase plástica (em N); alongamento no limite máximo, que é o valor de alongamento no ponto de maior carga na fase plástica (em $\mathrm{mm}$ ); rigidez, que corresponde à resistência passiva do músculo (em $\mathrm{N} / \mathrm{mm}$, para qualquer ponto da porção linear do gráfico); e resiliência, que representa a capacidade de absorver energia durante a fase elástica, isto é, até o limite de proporcionalidade (em Joules, J) (figura 1).

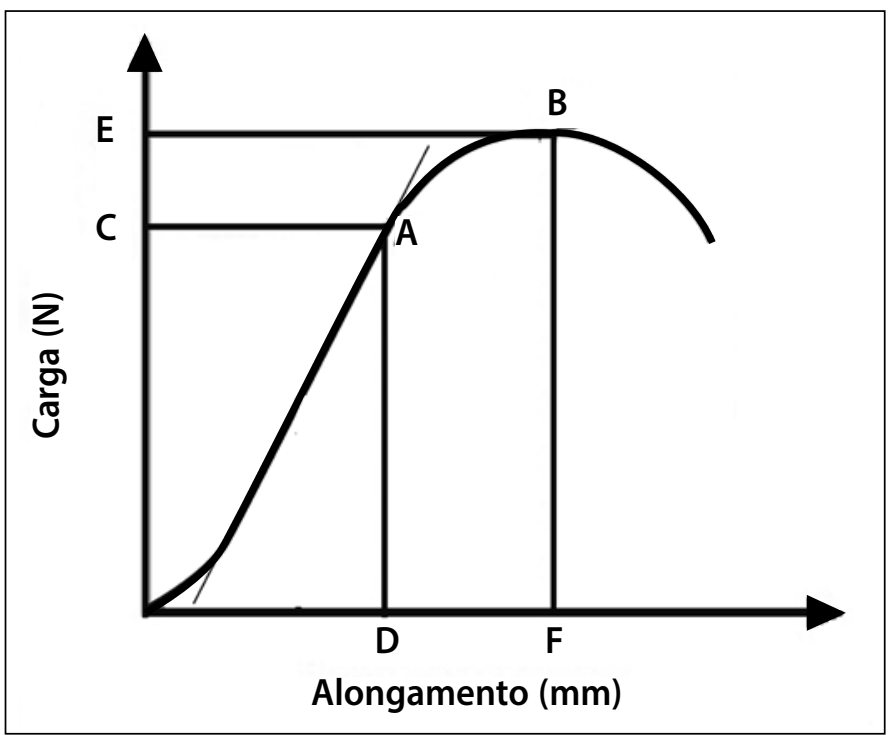

Figura 1. Representação esquemática da curva carga versus alongamento. Os limites elástico e plástico, bem como as propriedades mecânicas de interesse são mostrados: (A) limite de proporcionalidade, (B) limite máximo, (C) carga no limite de proporcionalidade, (D) alongamento no limite de proporcionalidade, (E) carga no limite máximo e (F) alongamento no limite máximo.

\section{Análise estatística}

A análise estatística dos dados foi inicialmente realizada com o teste Shapiro-Wilk, que mostrou distribuição normal em todas as variáveis. Em seguida, foi utilizado o teste de análise de variância (ANOVA), e para comparação entre os grupos foi empregado o teste post-hoc de Bonferroni. Grupos que não apresentaram valores significativamente diferentes foram marcados com a mesma letra minúscula (a), caso con- trário, foram marcados com letra minúscula distinta (b). Um valor de P menor que 0,05 foi considerado estatisticamente significativo. Todos os dados foram analisados a partir do pacote estatístico BioEstat ${ }^{\circledR}$ 2.0.

\section{RESULTADOS}

Ao todo, 30 músculos foram testados e os resultados expressos como média \pm desvio padrão (DP), para os três grupos, em termos de parâmetros biomecânicos.

\section{Carga no limite de proporcionalidade (CLP)}

O valor médio obtido após mensuração da CLP foi de 34,73 $\pm 9,40$ N para GC, 31,82 \pm 6,95 N para GL e 41,10 \pm 3,63 N para GLUST. Como pode ser observado na figura 2A, o grupo GLUST obteve valores significativamente maiores $(P=0,019)$ se comparados aos grupos $G C$ e $G L$.

\section{Alongamento no limite de proporcionalidade (ALP)}

Encontrou-se valores médios do ALP de 14,23 $\pm 3,73 \mathrm{~mm}, 13,94 \pm$ 1,96 mm e 15,43 \pm 1,29 mm para GC, GL e GLUST, respectivamente. Em relação a esta propriedade, não foram observadas diferenças estatisticamente significativas ( $P=0,6115$ ) entre os grupos experimentais (figura $2 B$ ).

\section{Carga no limite de máximo (CLM)}

Os valores médios da CLM para o GC foi de 39,60 \pm 8,02 N, enquanto para o GL foi de 34,68 \pm 7,38 N e para o GLUST, 44,92 \pm 3,90 N. $\mathrm{Na}$ análise simultânea dos grupos, foi observada diferença significativa $(P=0,0065)$ entre o grupo GLUST e os grupos GC e GL para esta propriedade mecânica (figura 2C).

\section{Alongamento no limite máximo (ALM)}

O valor médio do ALM para o GC foi de 15,95 2,64 mm; para o GL foi de 15,40 \pm 2,16 mm e finalmente, 17,17 \pm 1,67 $\mathrm{mm}$ para o GLUST. A propriedade mecânica ALM não apresentou diferença significativa entre os grupos, como mostra a figura 2D ( $P=0,1945)$.

\section{Rigidez (R)}

Os valores médios da rigidez foram 3,78 \pm 1,39 N/mm, 4,79 $\pm 2,19$ $\mathrm{N} / \mathrm{mm}$ e 4,99 \pm 0,58 N/mm para GC, GL e GLUST, respectivamente. Não foram observadas diferenças significativas $(P=0,1852)$ entre os grupos, no caso da rigidez (figura $2 \mathrm{E}$ ).

\section{Resiliência (Ur)}

O valor médio da resiliência para GC foi de 59,01 \pm 17,10 J, para GL obtemos 45,10 \pm 14,92 J e, para o GLUST, 63,14 \pm 3,83 J. Em relação a esta propriedade mecânica, foi constatada diferenças significativas $(\mathrm{P}=0,0140)$ na comparação entre os grupos (figura 2F).

\section{DISCUSSÃO}

Devido à alta incidência das lesões musculares, discussões sobre o seu tratamento vêm ganhando cada vez mais espaço nas áreas de reabilitação desportiva, traumatológica e ortopédica². Nesse contexto, a literatura cita o gastrocnêmio como um músculo que possui risco aumentado para lesões e rupturas, salientando os níveis extremos de carga aos quais está exposto 22 .

Estudos que visam elucidar técnicas de incremento do reparo tecidual, ou mesmo analisar a influência de certos fatores no surgimento de lesões, têm a mensuração das propriedades mecânicas do sistema muscular como parte imprescindível de estudo ${ }^{20,22}$. Tais análises são consideradas adequadas por fornecerem resultados relevantes sobre alterações e adaptações teciduais frente a diferentes demandas funcionais ${ }^{23}$. 


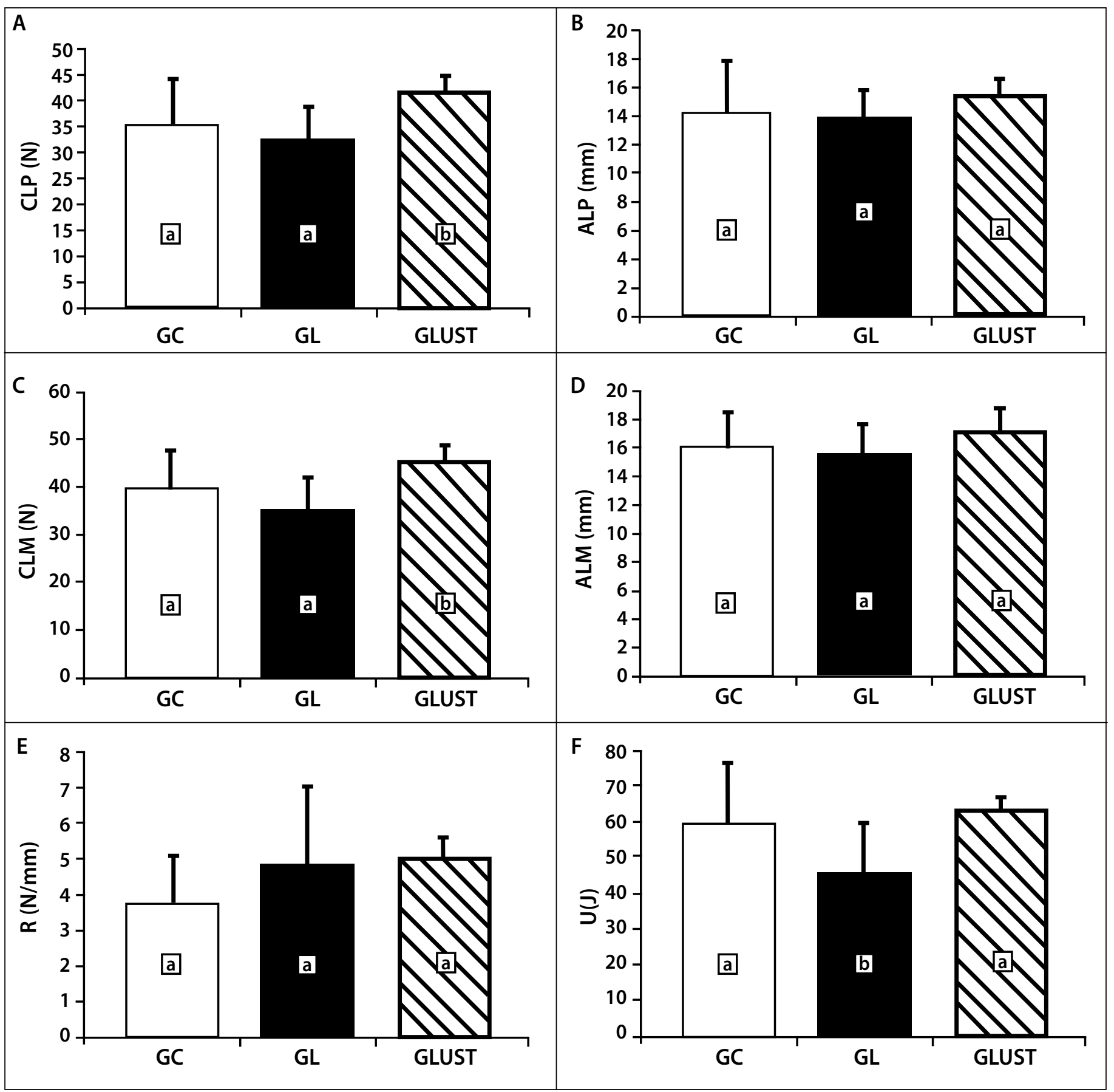

Figura 2. Valores médios \pm DP referentes às propriedades mecânicas avaliadas entre os grupos: carga no limite de proporcionalidade (A), alongamento no limite de proporcionalidade (B), carga no limite máximo (C), alongamento no limite máximo (D), rigidez (E) e resiliência (F). Grupos marcados com mesma letra minúscula (a) obtiveram médias estatisticamente iguais. $b \neq$ dos demais grupos.

Através do ensaio de tração realizado neste estudo, verificou-se redução significativa (23\%), do grupo GL em relação ao GC, na propriedade resiliência (Ur). Este resultado reflete uma menor capacidade do músculo lesionado em absorver energia em sua fase elástica (região linear da curva carga versus alongamento). Funcionalmente, tal fato implica em maior dissipação energética durante a deformação muscular, com baixo aproveitamento cinético e pior desempenho durante a locomoção ${ }^{24}$. Paralelamente, isto revela que o grupo GL sofreu alterações plásticas (lesões) com menores cargas, fato comum em distensões e rupturas ${ }^{25}$.

Constatou-se, ainda, acréscimo do grupo GLUST, em comparação ao GL, nas propriedades CLP (22\%), CLR (23\%) e Ur (28\%). Isto demonstra o efeito benéfico do UST na aceleração e na melhoria do processo de reparo muscular na fase de regeneração. Claramente, o UST aumentou a carga suportada nos extremos

teciduais elástico e plástico, e, ainda, promoveu maior absorção energética. Em situações reais, tais incrementos biomecânicos representam maior resistência a lesões frente à imposição de sobrecargas, bem como maior eficiência energética do sistema muscular.

Independentemente do tipo de lesão, o reparo da musculatura esquelética segue um padrão definido que consiste em três fases, parcialmente sobrepostas: destruição, reparo e remodelamento ${ }^{26}$. No presente estudo, a irradiação ultrassônica teve início 24 horas após a lesão experimental, período compreendido na fase de destruição. Considerando que o UST promove a neutralização de substâncias pró-inflamatórias e estimula o início da mioregeneração das fases subsequentes ${ }^{26,27}$, sua aplicação nos estágios iniciais do reparo muscular é crucial. 
Os resultados positivos encontrados nas propriedades CLP, CLM e Ur do grupo GLUST provavelmente se devem aos efeitos de incremento do tecido conjuntivo por parte do UST. Dentre os efeitos, destaca-se o estímulo ao aumento da síntese de proteínas como o colágeno e proteoglicanos ${ }^{26}$.

Como o ensaio foi realizado nove dias após a criolesão, o músculo dos animais lesionados encontrava-se num estágio de intensa proliferação celular, além de ativação e invasão de fibroblastos, responsáveis pela deposição de nova matriz colágena ${ }^{26}$. Desse modo, presume-se que o aumento expressivo do tecido fibroso nesta fase garantiu certa resistência e elasticidade aos músculos lesionados.

As propriedades ALP e ALM, relacionadas ao grau de estiramento suportado em pontos distintos da curva carga versus alongamento, não diferiram entre os grupos. Estes resultados corroboram com o trabalho de Oliveira et al. ${ }^{16}$, que também não verificou influências experimentais sobre o ALP e o ALM, entre os grupos equivalentes. A literatura apresenta outros trabalhos que, de maneira semelhante, não encontraram diferenças no alongamento suportado entre seus grupos experimentais, apesar de observarem diferenças nas demais propriedades ${ }^{17,20,28}$. Embora os autores não ofereçam explicação para este achado, é plausível admitir que, entre tecidos moles de dimensões reduzidas, a diferença entre medidas do grau de alongamento tende a se tornar pouco expressiva.

Não houve, ainda, diferença significativa entre os grupos na propriedade rigidez. Esta, nos termos discutidos aqui, é definida como a propriedade que representa a proporção direta entre a força aplicada e o alongamento observado no tecido, e pode ser percebida pelo grau de inclinação da região linear da curva carga versus alongamento ${ }^{29}$. Portanto, a ausência de incrementos ou decréscimos significativos pode ser parcialmente explicada como um reflexo do comportamento indistinto dos músculos nas propriedades ALP e ALM.

Adicionalmente, outra justificativa é oportuna neste caso: cada espécime muscular possui dimensões próprias e, assim, o formato da curva carga versus alongamento é diferente para cada um. Uma forma de contornar este obstáculo seria a normalização dos dados por meio das medidas de área de secção transversa e de comprimento inicial do músculo. A literatura, no entanto, reconhece que a obtenção de tais medidas oferece ainda maiores obstáculos ${ }^{30}$. O caráter destrutivo dos ensaios mecânicos confere grande dificuldade na mensuração adequada da área de secção transversa no local da lesão muscular ${ }^{19}$. Por estas razões, os procedimentos foram conduzidos de modo a avaliar os dados a partir da curva carga versus alongamento e não pela tensão versus deformação. Vale salientar que se buscou minimizar este problema com o controle do peso corporal dos animais, em todos os grupos. Antes e após o período experimental (nove dias), verificou-se estatisticamente que os grupos exibiram mesmo peso corporal.

\section{CONCLUSÃO}

Os resultados encontrados neste estudo demonstram que os músculos estimulados com UST apresentaram incrementos significativos nas propriedades mecânicas: carga no limite de proporcionalidade, carga no limite máximo e resiliência.

Considerando tais resultados, aliados ao achados da literatura, conclui-se que a terapia ultrassônica logo após a lesão favorece a função biomecânica do sistema muscular, sugerindo, em última instância, modificações em suas estruturas biológicas.

Para melhor fundamentação do emprego do UST nas propriedades mecânicas do tecido muscular, é importante a continuidade das pesquisas em médio e longo prazo, bem como o desenvolvimento de protocolos para determinação de parâmetros terapêuticos mais precisos e específicos para cada modelo experimental.

Todos os autores declararam não haver qualquer potencial conflito de interesses referente a este artigo.

\section{REFERÊNCIAS}

1. Järvinen TA, Järvinen TL, Kääriäinen M, Aärimaa V, Vaittinen S, Kalimo H, et al. Muscle injuries: optimising recovery. Best Pract Res Clin Rheumatol. 2007;2:317-31.

2. Assis LR. Laser de baixa intensidade $(830 \mathrm{~nm})$ na regeneração músculo tibial anterior em ratos. Dissertação (Mestrado). Universidade Federal de São Carlos, São Carlos, 2008. 36p.

3. Dalia RA, Guerino MR, Parizotto NA, Mello MAR, Luciano E. A influência do ultrassom e do treinamento físico sobre os triglicérides séricos e intramusculares em ratos diabéticos experimentais. Rev Bras Med Esporte. 2011;17:123-6

4. Speed CA. Therapeutic ultrasound in soft tissue lesions. Rheumatology (Oxford). 2001;40:1331-6.

5. Larsen A, Kristensen G, Thorlacius-Ussing O, Oxlund $H$. The influence of ultrasound on the mechanical properties of healing tendons in rabbits. Acta Orthop 2005;76:225-30.

6. Dogru H, Basaran S, Sarpel T. Effectiveness of therapeutic ultrasound in adhesive capsulitis. Joint Bone Spine. 2008;75:445-50.

7. Okita M, Nakano J, Kataoka H, Sakamoto J, Origuchi T, Yoshimura T. Effects of therapeutic ultrasound on joint mobility and collagen fibril arrangement in the endomysium of immobilized rat soleus muscle. Ultrasound Med Biol. 2009;35:237-44.

8. Ciena AP, Cunha NB, Moesch J, Mallmann JS, Carvalho AR, Moura PJ, et al. Efeitos do ultrassom terapêutico em modelo experimental de ciatalgia. Rev Bras Med Esporte. 2009;15:424-7.

9. Alfredo PP, Anaruma CA, Pião ACS, João SMA, Casarotto RA. Análise qualitativa dos efeitos da sonoforese com Arnica montana sobre o processo inflamatório agudo do músculo esquelético de ratos. Fisioter Pesq. 2008;15:273-9.

10. Wilkin LD, Merrick MA, Kirby TE, Devor ST. Influence of therapeutic ultrasound on skeletal muscle regeneration following blunt contusion. Int J Sports Med. 2004;25:73-7.

11. Mcbrier NM, Lekan JM, Druhan L, Devor ST, Merrick MA. Therapeutic ultrasound decreases mechano-growth factor messenger ribonucleic acid expression after muscle contusion injury. Arch Phys Med Rehabil. 2007;88:936-40.

12. Bertolini GRF, Barbieri CH, Mazzer N. Análise longitudinal de músculos sóleos, de ratos, submetidos a alongamento passivo com uso prévio de ultrassom terapêutico. Rev Bras Med Esporte. 2009;15:115-8.

13. Warden SJ, Komatsu DE, Rydberg J, Bond JL, Hassett SM. Recombinant human parathyroid hormone (PTH 1-34) and low intensity pulsed ultrasound have contrasting additive effects during fracture healing. Bone. 2009;44:485-94

14. Katano M, Naruse K, Uchida K, Mikuni-Takagaki Y, Takaso M, Itoman M, et al. Low intensity pulsed ultrasound accelerates delayed healing process by reducing the time required for the completion of endochondral ossification in the aged mouse femur fracture model. Exp Anim. 2011;60:385-95.
15. Shimano AC, Shimano MM. Ensaios tecnológicos de materiais biológicos. In: XVII Congresso Brasileiro de Engenharia Biomédica. Anais do XVII CEBEB. 2000;15-21p.

16. Oliveira FB, Shimano AC, Picado CHF. Ultra-som terapêutico e imobilização na reparação do trauma muscular. Acta Ortop Bras. 2009;17:167-70.

17. Polizello JC, Carvalho LC, Freitas FC, Padula N, Shimano AC, Mattiello-Sverzut AC. Propriedades mecânicas do músculo gastrocnêmio de ratas, imobilizado e posteriormente submetido a diferentes protocolos de alongamento. Rev Bras Med Esporte. 2009;15:195-9.

18. Robertson VJ, Baker KG. A review of therapeutic ultrasound: effectiveness studies. Phys Ther. 2001;81:1339-50

19. Demir H, Menku P, Kirnap M, Calis M, Ikizcelli I. Comparison of the effects of Laser, ultrasound, and combined laser + ultrasound treatments in experimental tendon healing. Lasers Surg Med. 2004;35:84-9.

20. Matheus JPC, Oliveira FB, Gomide LB, Milani JGPO, Volpon JB, Shimano AC. Efeitos do ultra-som terapêutico nas propriedades mecânicas do músculo esquelético após contusão. Rev Bras Fisioter. 2008;12:241-7.

21. Miyabara EH, Aoki MS, Moriscot AS. Cyclosporin A preferentially attenuates skeletal slow-twitch muscle regeneration. Braz J Med Biol Res. 2005b;38:559-63.

22. Järvinen MJ, Einola SA, Virtanen EO. Effect of the position of immobilization upon the tensile properties of the rat gastrocnemius muscle. Arch Phys Med Rehabil. 1992;73:253-7.

23. Caiozzo VJ. Plasicity of skeletal muscle phenotype mechanical consequences. Muscle Nerve. 2002;26:740-68.

24. Cutts A, Alexander RM, Ker RF. Ratios of cross-sectional areas of muscles and their tendons in a healthy human forearm. J Anat.1991;176:133-7.

25. Gould III JA. Biomecânica na terapia esportiva e ortopédica. In: Gould III JA. Fisioterapia na ortopedia e na medicina do esporte. São Paulo: Manole, 1993. p. 65-83.

26. Järvinen $T A$, Järvinen $T L$, Kääriäinen $M$, Kalimo $H$, Järvinen M. Muscle injuries: biology and treatment. Am J Sports Med. 2005;33:745-64.

27. Dyson M. Mechanism involved in therapeutic ultrasound. Physiotherapy. 1987;73:116-20.

28. Tomiosso TC, Nakagaki WR, Gomes L, Hyslop S, Pimentel ER. Organization of collagen bundles during tendon healing in rats treated with L-NAME. Cell Tissue Res. 2009;337:235-42.

29. Markolf KL, Kochan A, Amstutz HC. Measurement of knee stiffness and laxity in patients with documented absence of anterior cruciate ligament. J Bone Joint Surg Am. 1984;66:242-53.

30. Woo SL-Y, Abramowitch SD, Kilger R, Liang R. Biomechanics of knee ligaments: injury, healing, and repair. J Biomech. 2006:9:1-20 